\title{
New Power Minimization Techniques in Hybrid Distributed Antenna Systems with Orthogonal and Non Orthogonal Multiple Access
}

\author{
Antoine Kilzi, Joumana Farah, Charbel Abdel Nour, Catherine Douillard
}

\begin{abstract}
Distributed antenna systems have been proposed as a solution to supply the ever increasing capacity demands in next generation networks. This paper considers the power minimization problem in hybrid distributed antenna systems where antennas are supplied by various - low power and high power - energy sources. Antenna-specific power limits are considered and the problem is reformulated in this new hybrid context. The optimal power allocation problem is first formulated and solved in the orthogonal multiplexing scenario. Different resource allocations schemes based on this optimal power allocation are then proposed for the orthogonal and nonorthogonal multiplexing contexts. Simulation results illustrate the efficiency of the proposed algorithms and show the importance of non-orthogonal multiplexing in the reduction of the total transmission power, especially in hybrid antenna systems.
\end{abstract}

Index Terms-Distributed antenna systems, hybrid antenna deployment, waterfilling, power allocation, resource allocation.

\section{INTRODUCTION}

$\mathbf{R}$ ECENTLY Recently, distributed antenna systems (DAS) [1], [2] were predicted to be one of the main leveraging techniques in future mobile communication systems, since this technology offers several advantages over centralized antenna systems (CAS). The concept of DAS (also known as distributed base stations or DBS) consists in deploying the base station (BS) antennas throughout the cell, instead of having multiple antennas installed on a single tower at the cell center. Each antenna is referred to as remote radio head $(\mathrm{RRH})$. The aim of this configuration is to reduce the mean distance (and the path-loss) between users and their serving antennas, thus enabling the system to achieve a better reception quality. In addition, it makes it possible to benefit from the advantages of small cell networks while avoiding the problem of excessive hand-offs inherent to cell sectoring. In that regard, DAS emerges as a win-win trade-off between small cells and large sectored cells as it combines the best of both network architectures. Moreover, the spreading of antennas over each cell enables the system to better adapt to various user-network topologies, which strengthens the system's resilience to fading and provides a robust framework to combat inter-cell and intracell interference. These advantages have a green ecological

J. Farah is with the Department of Electricity and Electronics, Faculty of Engineering, Lebanese University, Roumieh, Lebanon (joumanafarah@ul.edu.lb).

A. Kilzi, C. Abdel Nour and C. Douillard are with Institut Mines-Telecom, CNRS UMR 6285 Lab-STICC, 29238 Brest, France, (email: antoine.kilzi@imt-atlantique.fr; charbel.abdelnour@imt-atlantique.fr; catherine.douillard@imt-atlantique.fr). impact as cells will be able to provide users with their requested services by utilizing the advantages of the network topology rather than resorting to an increase in the system transmission power. Therefore, DAS can greatly reduce local electromagnetic radiation and $\mathrm{CO}_{2}$ emissions of transmission systems.

One of the main challenges posed by DAS configurations lies in the elaboration of efficient resource allocation schemes that take advantage of the embedded spatial diversity, while allowing the implementation of efficient solutions with an affordable runtime complexity. The works in [3], [4] assess the ergodic capacity of DAS for two different transmission scenarios: selection diversity in which one of the RRHs is selected for transmitting a given signal, and blanket transmission where all antennas in the cell participate in each transmission. The results show that selection diversity achieves better capacity in DAS, while decreasing the number of information streams that need to be transferred among the involved RRHs, therefore reducing the signaling overhead [5].

The relative geographic proximity between the users and the antennas in DAS may give rise to more restrictive regulations on antenna power limits than before, in order to limit the electromagnetic field (EMF) exposure, especially in sensitive locations in dense urban areas (e.g. around hospitals, police stations, etc.). Therefore, in such hybrid configurations, certain antennas in the cell may have restrictive transmit power constraints (due to their geographical position, their powering source or their small size), while others have access to a much higher amount of available power. The development of procedures which can deal with such different restrictions goes along with the philosophy of future $5 \mathrm{G}$ communication in designing new smart networks that can dynamically adapt to various network demands and configurations. These procedures would also come in handy in situations where the operators use hybrid sources of energy to power the antennas deployed at different locations in the cell, including electric grids, local generators and various energy harvesting techniques.

Several works in DAS target the optimization of system energy efficiency (EE) with a power constraint on each RRH. In [6], the authors propose antenna selection as a means to maximize the $\mathrm{EE}$ of communication systems by successively activating antennas with a decreasing order in added efficiency. However, in a multi-carrier system where frequency selective channels are experienced by users, 
the possibility to use or not a particular antenna can be extended to each of the possible system subcarrier. Our work provides efficient solutions for the extended case of joint RRH/subcarrier selectivity. In [7], subcarrier assignment and power allocation (PA) are done in two separate stages. In the first stage, the number of subcarriers per RRH is determined, and subcarrier/RRH assignment is performed assuming initial equal power distribution. In the second stage, optimal PA relying on the sub-gradient method is performed to maximize the EE under the constraints of the total transmit power per $\mathrm{RRH}$, of the targeted bit error rate and of a proportionally-fair throughput distribution among active users. In [8], optimal $\mathrm{PA}$ is derived for EE maximization under antenna power constraints and proportionally fair user rates. Differently from [7], a single-variable non-linear equation needs to be solved. However the resource allocation problem in its integrality is not addressed since the joint subchannel and power allocation is not studied. The optimization techniques proposed in [6-8] for DAS are designed for the case of orthogonal multiple access (OMA). In other words, they allow the allocation of only one user per subcarrier.

Non-orthogonal multiple access (NOMA) has recently emerged as a promising multiple access technique to significantly improve the attainable spectral efficiency for $5 \mathrm{G}$ networks [9-11]. In Power-Domain NOMA (PD-NOMA), users collocated on the same time-frequency bloc are multiplexed in the power domain through the assignment of distinct power levels depending on their channel gain differences [12]. Successive Interference Cancellation (SIC) is carried out at the receiver side to recover the individual messages from the superimposed received signal.

In [13], we have introduced a set of techniques that allow the joint allocation of subcarriers and power, with the aim of minimizing the total power in CAS using NOMA techniques. Particularly, we showed that the most efficient method, from the power minimization perspective, consists in applying user pairing at a stage subsequent to single-user assignment, i.e. after applying OMA signaling at the first stage, instead of jointly assigning collocated users to subcarriers. In [14], we unveiled some of the potential of DAS systems combined with NOMA, in which we showed that under some specific subcarrier, users and powering antennas configurations, the two paired users on a subcarrier would be able to perform successive interference cancellation (SIC). Based on this property, we developed techniques for joint subcarrier and power allocation that aim at minimizing the total amount of power under user rate constraints in downlink NOMA. To the best of our knowledge, the problem of downlink power minimization in DAS networks with RRH power limits using NOMA has not been addressed yet. This problem is substantially different from the one in [14] since heavily loaded antennas are generally the most important players in minimizing the system power. Thus, setting power limits on some of them will necessarily raise the system power consumption. To address this problem, we derive the optimal PA scheme for OMA (given a predefined subcarrier assignment) and explore thoroughly its properties, prior to introducing complete resource allocation (RA) schemes that meet the system requirements based on the optimal PA, in both orthogonal and non-orthogonal scenarios.

The rest of this paper is organized as follows. Section II introduces the system model. Section III derives the optimal solution to the power allocation problem in OMA with hybrid distributed antenna systems (HDAS). Section IV proposes several new joint OMA resource allocation schemes based on the optimal PA, while Section V tackles the resource allocation problem in the NOMA HDAS scenario. In Section VI, a complexity analysis is provided to the different algorithms. Section VII presents the simulation results and Section VIII concludes the document.

\section{System DESCRIPTION AND PROBlem Formulation}

This study is conducted on a downlink system consisting of a total of $R$ RRHs uniformly positioned over a cell, where $K$ single-antenna mobile users are randomly deployed. In the current study, each RRH is supposed to be equipped with a single antenna. Therefore, in the sequel, the terms "RRH" and "antenna" will be used interchangeably. Among these $R$ RRHs, we consider a subset $\mathcal{R} \mathcal{L}=\left\{R L_{1}, R L_{2}, \ldots, R L_{F}\right\}$ of $F<R$ power-limited (or constrained) antennas having each a respective power limit $P_{m_{i}}, i=1, \ldots, F$, constituting the set $\mathcal{P}=\left\{P_{m_{1}}, P_{m_{2}}, \ldots, P_{m_{F}}\right\}$. The remaining $R-F$ RRHs have power limits much higher than those in $\mathcal{R} \mathcal{L}$, that is why their power constraints will not be considered in the following. These antennas constitute a set $\mathcal{R U}=$ $\left\{R U_{1}, R U_{2}, \ldots, R U_{R-F}\right\}$ of unconstrained antennas. All RRHs are connected to a single baseband unit (BBU) through high capacity optical fibers. Selection diversity [15] is assumed. The system bandwidth $B$ is equally divided into a total of $S$ subcarriers. Each user $k$ is allocated a set $\mathcal{S}_{k}$ of subcarriers by the BBU in a way to achieve a requested rate $R_{k, r e q}$ [bps]. From the set of $K$ users, a maximum of $m(n)$ users are chosen to be collocated on the $n^{\text {th }}$ subcarrier $(1 \leq n \leq S)$ using PDNOMA [12], [16]. Classical OMA signaling corresponds to the special case of $m(n)=1$. Also, in the sequel, we denote by DAS the system where $F=0$ (i.e. none of its RRHs has a power limitation), and by HDAS the case where $F \neq 0$.

The hybrid distributed antenna system is illustrated in Fig. 1 where orthogonal signaling is used to serve User 2 on subcarrier SC 3, and non-orthogonal signaling is used to serve Users 1, 2 and 3 on subcarriers SC 1 and SC 2 from both $\mathcal{R} \mathcal{L}$ and $\mathcal{R U}$ antennas.

Let $P_{k, n, r}$ be the power of user $k$ on subcarrier $n$, transmitted by RRH $r$, $\mathbb{H}$ the three-dimensional channel gain matrix with elements $h_{k, n, r}, 1 \leq k \leq K, 1 \leq n \leq S, 1 \leq r \leq R, k_{i}(n)$ the $i^{t h}$ multiplexed user on subcarrier $n, r_{i}(n)$ the antenna powering the signal of the $i^{t h}$ user on subcarrier $n$, and $\mathcal{S}\left(R L_{i}\right)$ the set of subcarriers powered by the $i^{t h}$ antenna in $\mathcal{R} \mathcal{L}$. At each receiver side, additive white gaussian noise is assumed with a power spectral density $N_{0}$, leading to the same average noise power $\sigma^{2}=N_{0} B / S$ on each subcarrier.

In classical PD-NOMA schemes, the same antenna is used to power the signals of collocated users on a subcarrier, which is the case of User 1 and User 2 on subcarrier SC 1 in Fig. 1. Users with high channel gains decode, remodulate and subtract 


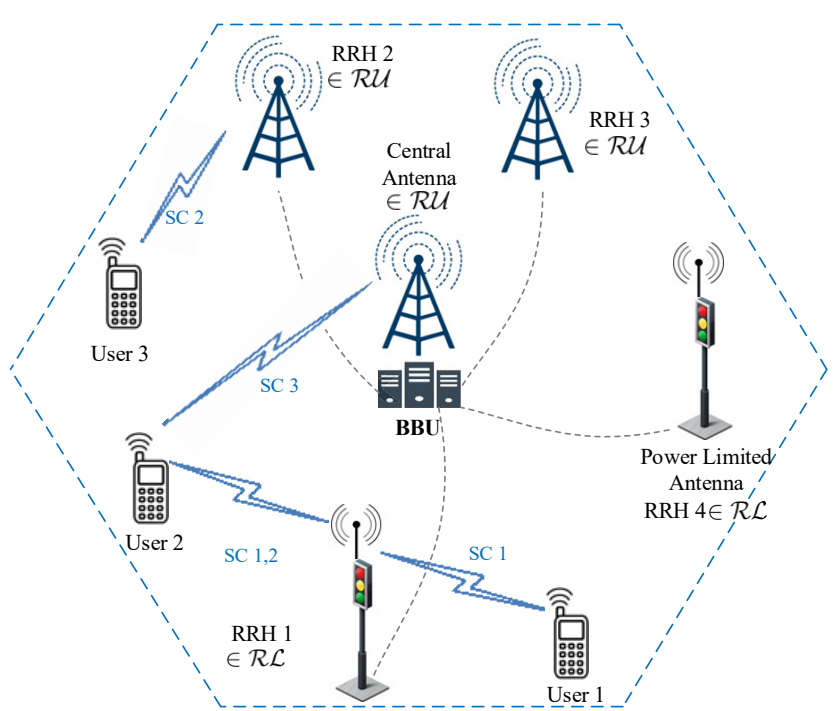

Figure 1: HDAS cell with two power-limited RRHs (RRH 1 and RRH 4)

the signals of weaker users, whereas weaker users suffer from the interference caused by the signals of stronger users. The interference cancellation at the level of the stronger users is guaranteed by allocating more power to UEs with poor channel conditions with respect to UEs with better channel conditions. In this study, we limit the number of collocated users to a maximum of 2 per subcarrier, which limits the SIC complexity at the receiver side at the cost of a negligible performance drop, compared to 3 collocated users, as it was shown in [12]. Therefore, the rate expressions and power multiplexing conditions of two collocated users $k_{1}$ and $k_{2}$ on subcarrier $n$ with $h_{k_{1}, n, r}>h_{k_{2}, n, r}$ and $r=r_{1}(n)=r_{2}(n)$ for classical PD-NOMA schemes are:

$$
\begin{gathered}
R_{k_{1}, n, r}=\frac{B}{S} \log _{2}\left(1+\frac{P_{k_{1}, n, r} h_{k_{1}, n, r}^{2}}{\sigma^{2}}\right) \\
R_{k_{2}, n, r}=\frac{B}{S} \log _{2}\left(1+\frac{P_{k_{2}, n, r} h_{k_{2}, n, r}^{2}}{P_{k_{1}, n, r} h_{k_{2}, n, r}^{2}+\sigma^{2}}\right) \\
P_{k_{2}, n, r}>P_{k_{1}, n, r}
\end{gathered}
$$

In our previous work [14], we showed that two users $k_{1}$ and $k_{2}$ power-multiplexed on a subcarrier $n$ and transmitted by two different RRHs $r_{1}(n)$ and $r_{2}(n)$ respectively, can perform mutual SIC, i.e. perform SIC simultaneously, if their channel gains verify:

$$
\frac{h_{k_{1}, n, r_{1}(n)}^{2}}{h_{k_{1}, n, r_{2}(n)}^{2}} \leq \frac{h_{k_{2}, n, r_{1}(n)}^{2}}{h_{k_{2}, n, r_{2}(n)}^{2}}
$$

In such cases, their theoretical throughputs and power multiplexing constraints are given by:

$$
\begin{aligned}
& R_{k_{1}, n, r_{1}(n)}=\frac{B}{S} \log _{2}\left(1+\frac{P_{k_{1}, n, r_{1}(n)} h_{k_{1}, n, r_{1}(n)}^{2}}{\sigma^{2}}\right) \\
& R_{k_{2}, n, r_{2}(n)}=\frac{B}{S} \log _{2}\left(1+\frac{P_{k_{2}, n, r_{2}(n)} h_{k_{2}, n, r_{2}(n)}^{2}}{\sigma^{2}}\right)
\end{aligned}
$$

$$
\frac{h_{k_{1}, n, r_{1}(n)}^{2}}{h_{k_{1}, n, r_{2}(n)}^{2}} \leq \frac{P_{k_{2}, n, r_{2}(n)}}{P_{k_{1}, n, r_{1}(n)}} \leq \frac{h_{k_{2}, n, r_{1}(n)}^{2}}{h_{k_{2}, n, r_{2}(n)}^{2}}
$$

The aim of the current study is to derive joint subcarrier and power allocation as well as user-pairing schemes that minimize the total transmit power while meeting the rate requirement of each user $\left(R_{k, \text { req }}\right)$ and the power limit constraints on the $\mathcal{R} \mathcal{L}$ antennas $(\mathcal{P})$. The introduction of power limit constraints on a subset of RRHs will lead to a more power consuming solution than the one obtained in [14], since the addition of any new constraint to an optimization problem may only result in the degradation of the solution's performance. Indeed, having power constraints on some antennas results in a power transfer from the constrained antennas to the unconstrained ones, in such a way that the requested rates remain satisfied for each user. Hence, minimizing the total transmit power of the system under the user rate and antenna power limit constraints translates into searching for the best "power transfer" scheme that minimizes the excess in power compared to the unconstrained DAS solution in [14]. Note that the number of constrained antennas $F$ shall not reach $R$, that is to say that at least one antenna has to remain unconstrained in order to guarantee the satisfaction of the requested rate for all users. The global optimization problem of user-subcarrier-RRH assignment and power allocation, taking into account the rate requirements, power limits, and NOMA power multiplexing constraints, can be formulated as:

$$
O \mathcal{P}_{1}:\left\{\mathcal{S}_{k}, P_{k, n, r}\right\}^{*}=\underset{\left\{\mathcal{S}_{k}, P_{k, n, r}\right\}}{\arg \min } \sum_{k=1}^{K} \sum_{\substack{n \in \mathcal{S}_{k} \\ r=r_{i}(n), \text { s.t. } k_{i}(n)=k}} P_{k, n, r},
$$

Subject to :

$$
\begin{aligned}
& \sum_{\substack{n \in \mathcal{S}_{k} \\
k_{i}, n, r_{i}(n)}}=R_{k, r e q}, \forall k, 1 \leq k \leq K \\
& \text { s.t. } k_{i}(n)=k, i=\{1,2\} \\
& \sum_{n \in \mathcal{S}\left(R L_{i}\right)} P_{k_{i}(n), n, R L_{j}} \leq P_{m_{i}}, \forall j, 1 \leq j \leq F \\
& k=k_{i}(n) \text { s.t. } r_{i}(n)=R L_{j}
\end{aligned}
$$

This problem involves set selection as well as continuous variable optimization. Hence its mixed-integer non-convex nature justifies the introduction of suboptimal schemes. The joint subband and power allocation techniques developed in [14] consisted of successively applying orthogonal and nonorthogonal signaling. Therefore, in this work, we first derive new efficient techniques for resolving the power minimization problem in the OMA context for a predefined subcarrier allocation (Section III), while taking into account constraints $(3,4)$ for $m(n)=1$. This optimal PA is incorporated in different resource allocation methods in Section IV, for the case of OMA with HDAS. Then, the case of NOMA with HDAS is studied in Section V. 


\section{Optimal Power Allocation for OMA HDAS}

In this section, for a predefined subcarrier allocation, the problem of optimal power allocation in the case of OMA transmission under user rate requirements and power limited antennas is studied first. The solution is then explored in details in order to provide an understanding of the behavior of the underlying system, identifying the key parameters enabling the proposal of reliable resource allocation schemes.

In the orthogonal scenario, every subcarrier $n$ is allocated to one user and one antenna at most, referred to as $k(n)$ and $r(n)$ respectively. The optimal PA scheme, for a predefined subcarrier allocation scheme, is cast as the solution to the following problem:

$$
\mathcal{O P}_{2}: \min _{\left\{P_{k, n, r}\right\}} \sum_{k=1}^{K} \sum_{n \in \mathcal{S}_{k}} P_{k, n, r(n)}
$$

Subject to:

$$
\begin{gathered}
\sum_{n \in \mathcal{S}_{k}} \frac{B}{S} \log _{2}\left(1+\frac{P_{k, n, r(n)} h_{k, n, r(n)}^{2}}{\sigma^{2}}\right)=R_{k}, \forall k, 1 \leq k \leq K \\
\sum_{n \in \mathcal{S}\left(R L_{i}\right)} P_{k(n), n, R L_{i}} \leq P_{m_{i}}, \forall i, 1 \leq i \leq F
\end{gathered}
$$

The problem in hand can be solved by means of standard convex optimization techniques. Its Lagrangian is given by:

$$
\begin{aligned}
& L\left(P_{k, n, r}, \lambda_{k}, \alpha_{i}\right)=-\sum_{k=1}^{K} \sum_{n \in \mathcal{S}_{k}} P_{k, n, r(n)} \\
&+\sum_{k=1}^{K} \lambda_{k}\left(R_{k}-\sum_{n \in \mathcal{S}_{k}} \frac{B}{S} \log _{2}\left(1+\frac{\left.P_{k, n, r(n)} h_{k, n, r(n)}^{2}\right)}{\sigma^{2}}\right)\right) \\
&+\sum_{i=1}^{F} \alpha_{i}\left(P_{i}-\sum_{n \in \mathcal{S}\left(R L_{i}\right)} P_{k(n), n, R L_{i}}\right)
\end{aligned}
$$

where $\lambda_{k}$ and $\alpha_{i}$ represent the Lagrangian multipliers relative to the rate and power constraints respectively.

The corresponding Karush-Kuhn-Tucker (KKT) conditions are:

$$
\begin{cases}\nabla L\left(P_{k, n, r(n)}^{*}, \lambda_{k}^{*}, \alpha_{i}^{*}\right)=0 & \\ \sum_{n \in \mathcal{S}_{k}} \frac{B}{S} \log _{2}\left(1+\frac{P_{k, n, r(n)} h_{k, n, r(n)}^{2}}{\sigma^{2}}\right)=R_{k}, & \forall k, 1 \leq k \leq K \\ \sum_{n \in \mathcal{S}\left(R L_{i}\right)} P_{k(n), n, R L_{i}} \leq P_{i}, & \forall i, 1 \leq i \leq F \\ \left.\alpha_{i} \sum_{n \in \mathcal{S}\left(R L_{i}\right)} P_{k(n), n, r(n)}-P_{i}\right)=0, & \forall i, 1 \leq i \leq F \\ \alpha_{i} \geq 0, & \forall i, 1 \leq i \leq F\end{cases}
$$

The expressions of the partial derivatives of $L$ with respect to the different power variables depend on the type of RRH powering the subcarrier. After differentiation and some manipulations detailed in Appendix A, we obtain the following expressions for the subcarrier powers in terms of $\lambda_{k}$ and $\alpha_{i}$ :

$$
\begin{gathered}
P_{k, n, r(n)}=\frac{m_{k}}{\left(1+\alpha_{i}\right)}-\frac{\sigma^{2}}{h_{k, n, r(n)}^{2}}, \forall n \in \mathcal{S}\left(R L_{i}\right) \\
P_{k, n, r(n)}=m_{k}-\frac{\sigma^{2}}{h_{k, n, r(n)}^{2}}, \forall n \notin \bigcup_{i=1}^{F} \mathcal{S}\left(R L_{i}\right)
\end{gathered}
$$

where $m_{k}=-B \lambda_{k} / S \ln 2$. If $O \mathcal{P}_{1}$ was considered without the power constraints (8), the solution of the PA problem would revert to the classical case of power minimization with user-specific rate constraints. In such a case, the solution would be the well-known waterfilling procedure [13], [17], and the waterline $m_{k}$ of user $k$ would be the same for all the subcarriers of the user. However, in a HDAS, the waterline becomes specific to the separate classes of subcarriers (grouped according to the transmitting antenna) allocated to the user. More specifically, equations (10) and (11) show that, for every user $k$, a specific waterlevel $m_{k i} \triangleq m_{k} /\left(1+\alpha_{i}\right)$ is assigned for every subset of subcarriers allocated to $k$ on the constrained antenna $R L_{i}$, whereas the remaining subcarriers of $k$ that are not powered by a constrained RRH share a common waterlevel $m_{k}$. Furthermore, all the waterlevels of the user $k$, corresponding to its powering RRHs, are related to $m_{k}$ by the factors $\left(1+\alpha_{i}\right)$.

Replacing the power variables by their expressions from (10) and (11) in the rate constraints (7), and applying some manipulations to the rate constraints yield the following forms, for each user $k$ :

$$
\begin{array}{r}
\sum_{n \in \mathcal{S}_{k} \cap \overline{\mathcal{S}(\mathcal{R} \mathcal{L})}} \log _{2}\left(m_{k} \frac{h_{k, n, r(n)}^{2}}{\sigma^{2}}\right)+\sum_{i=1}^{F} \sum_{n \in \mathcal{T}_{k i}} \log _{2}\left(\frac{m_{k} h_{k, n, r(n)}^{2}}{\left(1+\alpha_{i}\right) \sigma^{2}}\right)=\frac{R_{k} S}{B} \\
\sum_{n \in \mathcal{S}_{k} \cap \overline{\mathcal{S}(\mathcal{R} \mathcal{L})}} \log _{2}\left(m_{k} \frac{h_{k, n, r(n)}^{2}}{\sigma^{2}}\right)+\sum_{n \in \cup_{i=1}^{F} \mathcal{T}_{k i}} \log _{2}\left(m_{k} \frac{h_{k, n, r(n)}^{2}}{\sigma^{2}}\right) \\
-\sum_{i=1}^{F}\left|\mathcal{T}_{k i}\right| \log _{2}\left(1+\alpha_{i}\right)=\frac{R_{k} S}{B}
\end{array}
$$

where $\mathcal{S}(\mathcal{R} \mathcal{L}) \triangleq \cup_{i=1}^{F} \mathcal{S}\left(R L_{i}\right)$ is the set of all subcarriers powered by a power constrained antenna, and $\mathcal{T}_{k i} \triangleq \mathcal{S}_{k} \cap \mathcal{S}\left(R L_{i}\right)$ is the set of subcarriers allocated to user $k$ and powered by $R L_{i}$. Consequently, we obtain:

$$
\sum_{n \in \mathcal{S}_{k}} \log _{2}\left(m_{k} h_{k, n, r(n)}^{2} / \sigma^{2}\right)-\sum_{i=1}^{F}\left|\mathcal{T}_{k i}\right| \log _{2}\left(1+\alpha_{i}\right)=\frac{R_{k} S}{B}
$$

Therefore, $m_{k}$ can be written as:

$$
\begin{gathered}
m_{k}=\left(\frac{2^{\frac{R_{k} S}{B}}}{\prod_{n \in \mathcal{S}_{k}} h_{k, n, r(n)}^{2} / \sigma^{2}} \prod_{i=1}^{F}\left(1+\alpha_{i}\right)^{\left|\mathcal{T}_{k i}\right|}\right)^{1 /\left|\mathcal{S}_{k}\right|} \\
m_{k}=W_{k} \prod_{i=1}^{F}\left(1+\alpha_{i}\right)^{\frac{\left|\mathcal{T}_{k i}\right|}{\left|\mathcal{S}_{k}\right|}}
\end{gathered}
$$

As shown in Appendix $\mathrm{B}, W_{k}$ is the common waterline that user $k$ would have had in a "classical" waterfilling scheme, i.e. if the power constraints on $\mathcal{R} \mathcal{L}$ were not taken into account $\left(m_{k}=W_{k}\right.$ when $\left.\alpha_{i}=0, \forall i\right)$. Note that if user $k$ has all of 
its subcarriers powered by non-constrained antennas $\left(\mathcal{T}_{k i}=\emptyset\right.$, $1 \leq i \leq F$ ), or if a unique constrained RRH is exclusively serving the user $\left(\exists i \in\{1, \ldots, F\} \backslash \mathcal{T}_{k i}=\mathcal{S}_{k}\right)$, its corresponding waterline is not affected by the power correction and it is unique over the subcarrier set $\mathcal{S}_{k}$. The first case is proven in Appendix B, whereas the second can be directly deduced from (12) and (10).

A third possibility for having unique waterlines (per user) resides in a system where the classical waterfilling solution abides by (8). Indeed, if all the $\alpha_{i}$ variables were null, the resulting Lagrangian would not account for the power constraints (8), hence the solution would be a simple userbased waterfilling (if $\alpha_{i}=0, m_{k}=\frac{m_{k}}{\left(1+\alpha_{i}\right)}=W_{k}$ which results in a uniform waterlevel over all the subcarriers of the user). Furthermore, we can conclude that if an initial resource allocation technique verifies the constraints on the antennas assuming a user-based waterfilling, the latter is indeed the best PA solution for the given subcarrier allocation. On the other hand, the proposed optimal power allocation technique can be applied in association with any subcarrier allocation scheme that constitutes a solution to problem $O \mathcal{P}_{1}$.

Solving the optimization problem reduces to determining the $F$ Langrangian variables $\alpha_{i}$ relative to the power constraints. By replacing (12) into (10), the power constraint (8), corresponding to $\alpha_{i} \neq 0$ in the KKT conditions, for the $i^{t h}$ antenna $R L_{i}$ in $\mathcal{R} \mathcal{L}$, can be written as:

$$
\sum_{n \in S\left(R L_{i}\right)}\left(\frac{W_{k(n)} \prod_{j=1}^{F}\left(1+\alpha_{j}\right)^{\frac{\left|T_{k(n) i}\right|}{\left|s_{k(n)}\right|}}}{\left(1+\alpha_{i}\right)}-\frac{\sigma^{2}}{h_{k(n), n, r(n)}^{2}}\right)=P_{m_{i}}
$$

Equation (13) consists of $F$ non-linear equations with unknowns $\alpha_{i}$. In the sequel, the case of a single powerlimited antenna $(F=1)$ is considered first in order to provide a clear analysis of the hybrid system behavior. Then, the generalized study for higher values of $F$ is developed.

For the special case of a single power-limited antenna, we simply denote by $R L$ the concerned RRH and $\alpha$ the Lagrangian variable relative to the corresponding power constraint. For each user, we can identify at most two sets of subcarriers and thus two waterlevels which are related by the factor $(1+\alpha)$. The waterlevel of the subcarrier set that is not powered by the constrained antenna $R L$ is obtained from (12) as:

$$
m_{k}=(1+\alpha)^{\frac{\left|\mathcal{T}_{k}\right|}{\left|\mathcal{S}_{k}\right|}} W_{k}
$$

Equation (14) shows how the introduction of the constraint on one of the antennas affects the PA scheme, compared to the non-constrained case: since $\left|\mathcal{T}_{k}\right| /\left|\mathcal{S}_{k}\right| \leq 1$, and $\alpha>0$, the waterline of the subcarriers in $\mathcal{T}_{k}$ decreases with respect to $W_{k}$ (since $\left.m_{k} /(1+\alpha)<W_{k}\right)$, while that of the subcarriers in $\mathcal{S}_{k} \cap \overline{\mathcal{T}_{k}}$ increases (since $m_{k}>W_{k}$ ). This behavior is depicted in Fig. 2.

When the PA solution of the unconstrained problem does not respect (8), i.e. a power correction is necessary using (10) and (11), the rate transfer from the constrained antenna to the unconstrained ones translates into an unbalanced power

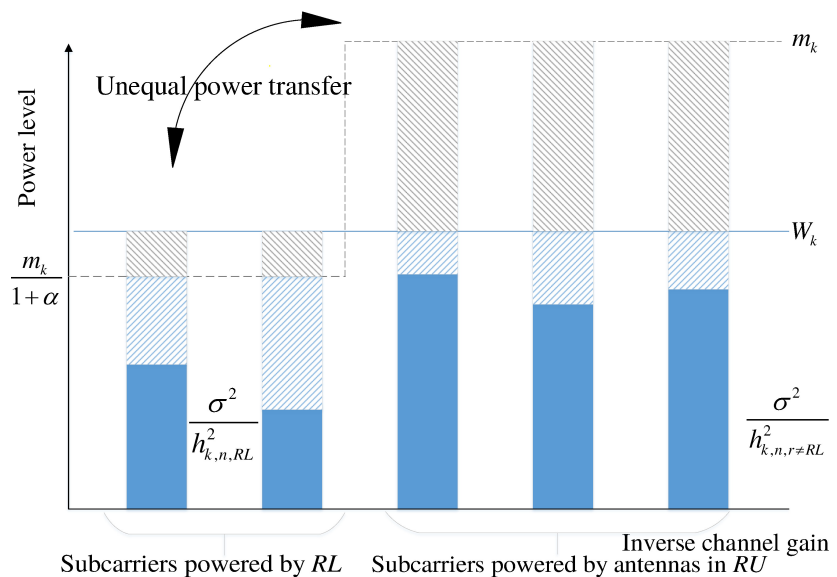

Figure 2: Power pouring diagram for a user $k$ after power correction

transfer from antenna $R L$ to the other antennas. Therefore, $\alpha$ could be seen as the deviation factor from the unconstrained problem (classical user-based waterfilling). A greater value of $\alpha$ translates into a greater deterioration of the performance of the solution towards that of the unconstrained problem (i.e. a more important increase of the total power in HDAS compared to DAS).

When $F=1$, the system of equations in (13) reduces to a single equation with a unique unknown $\alpha$ :

$$
\sum_{n \in \mathcal{S}(R L)}\left(W_{k(n)}(1+\alpha)^{\frac{\left|\mathcal{\tau}_{k(n)}\right|}{\left|s_{k(n)}\right|}-1}-\frac{\sigma^{2}}{h_{k(n), n, r(n)}^{2}}\right)=P_{m}
$$

There is no a priori guarantee for the existence of a solution to (15). An example of a situation with no solution is when every user served by $R L$ is exclusively linked to $R L$. Keeping in mind that such users are not affected by the power correction, if their total power consumption is greater than $P_{m}$, then no power assignment could, at the same time, verify the antenna power constraint and provide the users the rates they are requesting. Therefore, it is of interest to assess the feasibility of a proposed subcarrier allocation before proceeding to the resolution of (15) through a numerical solver. By isolating the $\alpha$ terms from the others, (15) takes the following form:

$$
\begin{gathered}
\sum_{\substack{n \in \mathcal{S}(\mathcal{R} \mathcal{L}), \mathcal{T}_{k(n)} \neq \mathcal{S}_{k(n)}}} W_{k(n)}(1+\alpha)^{\frac{\left|\mathcal{T}_{k(n)}\right|}{\left|\mathcal{S}_{k(n)}\right|}}=P_{m}-\sum_{\substack{n \in \mathcal{S}(R L), \mathcal{T}_{k(n)}=\mathcal{S}_{k(n)}}} W_{k(n)}+\sum_{n \in \mathcal{S}(R L)} \frac{\sigma^{2}}{h_{k(n), n, r(n)}^{2}} \\
A(\alpha)=C
\end{gathered}
$$

The first sum is a function of $\alpha$, hence the notation $A(\alpha)$. It includes all the subcarriers in $\mathcal{S}(R L)$ belonging to users who are served by at least one non-constrained antenna. $C$ is constant (for a fixed subcarrier allocation) and accounts for: the power limit, the waterlines relative to the subcarriers of the users exclusively served by $R L$, and finally, the inverse channel gains of all subcarriers powered by $R L$. In order to have a solution, $C$ needs to belong to the image of $A$ when $\alpha$ spans the positive real axis. However, $A(\alpha)$ is a polynomial 
with negative, fractional exponents and positive coefficients. Thus, it is a strictly decreasing function of $\alpha$, its co-domain is ]0; $A(0)$ ]. Therefore, the condition that guarantees the existence of a solution is: $0<C \leq A(0)$.

Proposition 1. If the system requires a power correction, $C$ will be necessarily smaller than $A(0)$

Proof. The left hand side of (15) is the power on $R L$ for a given value of $\alpha$. When $\alpha=0$, and since the system requires correction, this power is the actual power of $R L$ before any power correction takes place. This value is greater than $P_{m}$, i.e. $\sum_{n \in \mathcal{S}(R L)}\left(W_{k(n)}-\sigma^{2} / h_{k(n), n, r(n)}^{2}\right) \geq P_{m}$. By setting $\alpha$ to 0 in (16), we directly obtain $A(0)-C \geq 0$, i.e. $A(0) \geq C$ which concludes our proof.

As a result, the existence of a solution is only conditioned by $C$ being strictly positive. Finally, the uniqueness of the solution is an immediate result of the monotonic nature of function $A$.

\section{Resource Allocation fOr HDAS USING OMA}

Having established the main properties and conditions of the optimal power allocation, we now seek efficient resource allocation schemes that meet the rate and power limit requirements while minimizing the total power. In the following, two different approaches are proposed to resolve $O \mathcal{P}_{1}$ in the OMA context: OMA-HDAS and OMA-HDAS-Realloc. They both aim at determining the subcarrier and power allocation schemes that minimize the overall power, while guaranteeing the power and rate allocation constraints. OMA-HDAS take into consideration the antenna power constraints at the end of the algorithm while OMA-HDAS-Realloc accounts for the loading of the constrained antennas throughout the algorithm.

\section{A. OMA-HDAS}

Following the discussion in Proposition 1, a success guaranteed RA scheme is one that ensures the positivity of $C$. One sufficient condition for such solutions resides in removing the negative term from the right hand side of (16). This is achieved by imposing:

$$
\left|\mathcal{T}_{k}\right|<\left|\mathcal{S}_{k}\right|, \forall k
$$

In other terms, a sufficient condition for a success guaranteed RA scheme is to have every user served by $\mathcal{R} \mathcal{L}$ allocated at least one subcarrier powered by a non-constrained antenna. This is ensured in the Worst-Best-H (WBH) phase in Algorithm 1. Then, similarly to the techniques in [14], the power minimization strategy employed in this work follows a greedy approach where the most power consuming user is selected to be assigned the subcarrier-RRH pair that minimizes its total power. Finally, at the end of the allocation phase, the state of the $\mathcal{R} \mathcal{L}$ antennas are checked: if a power level higher than the imposed limit is detected, then the optimal power allocation is applied to perform power correction.

The details of the algorithm are presented hereafter, where $\mathcal{U}_{0}$, $\mathcal{S}_{p}$, and $\mathcal{R}$ represent respectively the sets of active users, free subcarriers, and the set of antennas that will be used to power the signals.

$\overline{\text { Algorithm 1 OMA-HDAS }}$
Initialization: $\mathcal{U}_{0}=\{1,2, \ldots, K\} ; \mathcal{S}_{k}=\emptyset, \forall k \in \mathcal{U}_{0} ; \mathcal{S}_{p}=\{1,2, \ldots, S\}$

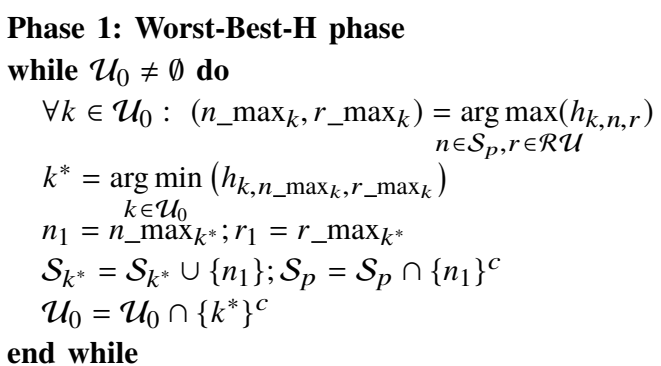

PA for each user on its allocated subcarrier so as to reach its requested rate

\section{Phase 2: Subcarrier-RRH assignment}

Initialization: $\mathcal{U}_{0}=\{1,2, \ldots, K\} ; \mathcal{R}=\mathcal{R} \mathcal{U} \cup \mathcal{R} \mathcal{L}$

$P_{k}:$ total power of user $k$,

$$
\begin{aligned}
& P_{R L_{i}}: \text { total power over RRH } R L_{i} \in \mathcal{R} \mathcal{L} \\
& \text { while } \mathcal{U}_{0} \neq \emptyset \& \mathcal{S}_{p} \neq \emptyset \text { do } \\
& k^{*}=\underset{k \in \mathcal{U}_{0}}{\arg \max }\left(P_{k}\right) \\
& \left(n^{*}, r^{*}\right)=\underset{n \in \mathcal{S}_{p}, r \in \mathcal{R}}{\arg \max }\left(h_{k, n, r}\right) \\
& \text { Estimate the power reduction } \Delta P \\
& \text { if } \Delta P<-\rho \\
& \quad \mathcal{S}_{k^{*}}=\mathcal{S}_{k^{*}} \cup\left\{n^{*}\right\} ; \mathcal{S}_{p}=\mathcal{S}_{p} \cap\left\{n^{*}\right\}^{c} \\
& \quad P_{k^{*}}=P_{k^{*}}+\Delta P \\
& \quad \text { Update } P_{\mathcal{R} \mathcal{L}} \\
& \text { else } \\
& \quad \mathcal{U}_{0}=\mathcal{U}_{0} \cap\left\{k^{*}\right\}^{c} \\
& \text { endif } \\
& \text { end while }
\end{aligned}
$$

\section{Phase 3}

if $\exists i \in\{1, \ldots, F\} / P_{R L_{i}}>P_{m}$

Apply the power correction using (13), (10) and (11) end

The power variation $\Delta P$ results from the decrease of the waterline level following the allocation of a new subcarrier to the user, and is calculated according to the low-complexity method introduced in [13] and [14]. Only effective subcarrier allocations are accepted, that is allocations leading to meaningful power variations where $\Delta P<-\rho$. $\rho$ is a power threshold chosen in such a way to strike a balance between power efficiency and spectrum occupancy. It is chosen according to the policy detailed in [18].

The main advantages of OMA-HDAS are its relative simplicity and its guarantee for providing a solution to the system. However, separating the subcarrier-RRH assignment from the correction phase is far from optimal since a beneficial subcarrier-RRH allocation on $\mathcal{R} \mathcal{L}$ in the first two phases of Algorithm 1 may turn out to be penalizing after power correction. In fact, when no special care is given in the subcarrier allocation to account for the subsequent power correction, a great load may result on $\mathcal{R} \mathcal{L}$, rendering the toll of the correction unacceptable. For instance, the power increase incurred by the power correction could be such that turning 
off the constrained antennas (or a subset of them) and then applying the simple power minimization procedure in [14] would be more profitable. To solve this problem, we propose in OMA-HDAS-Realloc a new approach for solving $O \mathcal{P}_{1}$.

\section{B. OMA-HDAS-Realloc}

To overcome the aforementioned drawbacks of OMAHDAS, we seek an RA scheme that can systematically outperform the trivial solution that consists in turning off the constrained antennas. For this purpose, the current algorithm applies two phases prior to the power correction. First, the trivial solution is applied: the constrained antennas are virtually shut off and the OMA power minimization technique (Phases 1 and 2 from Algorithm 1) is applied. In the second phase, the solution is enhanced by gradually moving some subcarriers from $\mathcal{R U}$ to $\mathcal{R} \mathcal{L}$, thus exploiting the better links available through the $\mathcal{R} \mathcal{L}$ antennas. To do so, the most power demanding user $k$, whose power can still be reduced, is selected and its subcarriers are considered for a potential reallocation.

To determine the subcarrier whose reallocation is the most profitable to user $k$, let us consider $r_{\text {old }}=r(n)$ the antenna powering the subcarrier $n$ before reallocation, and $r_{\text {new }}$ the candidate RRH considered for reallocation. To simplify the analysis, their corresponding channel gains are denoted by $h_{\text {old }}=h_{k, n, r_{\text {old }}}$ and $h_{n e w}=h_{k, n, r_{n e w}}$ respectively.

Proposition 2. The subcarrier leading to the greatest power decrease for user $k$ is the one having the highest ratio $h_{\text {new }} / h_{\text {old }}$, and the selected RRH is the one providing the largest channel gain on the selected subcarrier.

The proof of Proposition 2 is provided in Appendix C. When $h_{\text {new }} / h_{\text {old }}>1$ the reallocation is applied and the total power and waterline level of the user are updated. This reallocation process is carried out until leading to an excess in power over every antenna in $\mathcal{R} \mathcal{L}$. Note that if a reallocation renders any user without any sole subcarriers powered by $\mathcal{R} \mathcal{U}$, then this reallocation is rejected in order to guarantee the existence of a solution to the system according to (17). The details of OMARealloc-PC are presented in Algorithm 2.

\begin{tabular}{l}
\hline Algorithm 2 OMA-HDAS-Realloc \\
$\begin{array}{l}\text { Initialization: } \mathcal{U}_{0}=\{1,2, \ldots, K\} ; \mathcal{S}_{k}=\emptyset, \forall k \in \mathcal{U}_{0} ; \mathcal{S}_{p}= \\
\{1,2, \ldots, S\}\end{array}$
\end{tabular}

Phase 1:

WBH phase followed by Phase 2 of Algorithm 1 but with $\mathcal{R}=\mathcal{R} \mathcal{U}$

Phase 2: Subcarrier reallocation

Initialization: $\mathcal{U}_{0}=\{1,2, \ldots, K\} ; ; \mathcal{R}=\mathcal{R} \mathcal{L}$

$\mathcal{S}_{k}^{\mathcal{R U}} \triangleq \mathcal{S}_{k} \cap \overline{\mathcal{S}(\mathcal{R L})}$ : set of subcarriers of user $k$ powered by an antenna in $\mathcal{R U}$.

while $\mathcal{U}_{0} \neq \emptyset \& \mathcal{R} \neq \emptyset$ do

$k^{*}=\arg \max \left(P_{k}\right)$

$$
\left(n^{*}, r^{*}\right)=\underset{n \in \mathcal{S}_{k^{*}}^{\mathcal{R U}}, r \in \mathcal{R}}{\arg \max }\left(\frac{h_{k^{*}, n, r}}{h_{k^{*}, n, r(n)}}\right) / / \text { with } r=r_{\text {new }}, r(n)=r_{\text {old }}
$$

Estimate $\Delta P$ according to (23) in Appendix $\mathrm{C}$

if $\Delta P<-\rho$

$$
P_{k^{*}}=P_{k^{*}}+\Delta P
$$

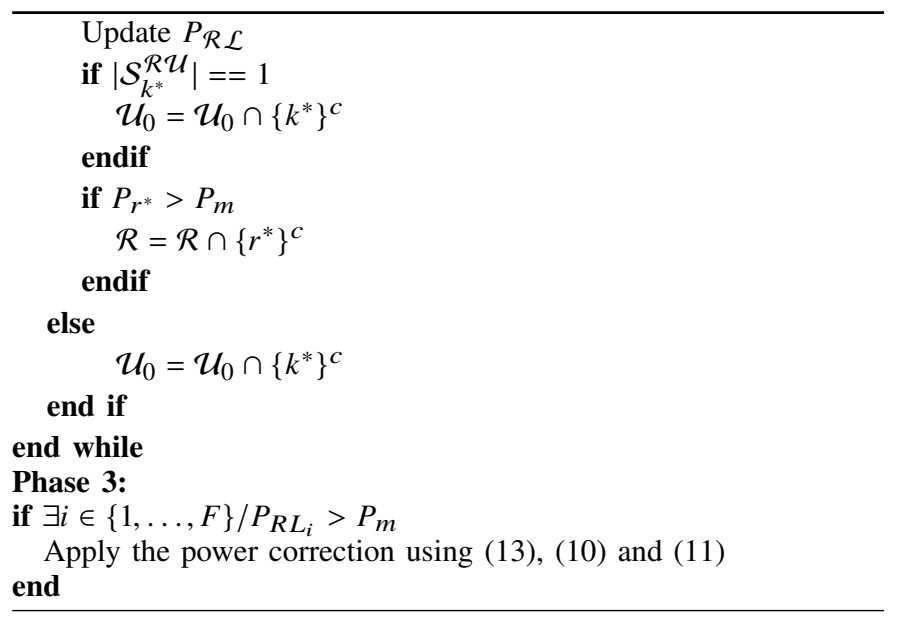

\section{Resource Allocation for HDAS using NOMA}

To further reduce system power, NOMA is applied on top of OMA. For this purpose, the user pairing scheme introduced in [14] (Mut\&SingSIC) is adapted to account for the antenna power limits. The allocation technique starts with an uncorrected version of the proposed solutions for OMA in Section IV. Then, the algorithm tries to pair users in order to reduce system power prior to applying a power correction at a final stage. As in [13] and [14], each time a pairing is performed on a subcarrier, the users powers on this subcarrier are kept unvaried for the subsequent allocation stages. In other words, they will not undergo any further modifications in the succeeding PA steps, in order to avoid complex chains of modifications. Due to the power multiplexing constraints of mutual and single SIC subcarriers $(5,6)$, the optimal PA described in Section IV for OMA can not be directly applied to the non-orthogonal context. Indeed, since the power allocated to multiplexed subcarriers is constant until the end of the pairing phase, the power correction has to be carried out on the sole subcarriers only (subcarriers occupied by a unique user). Moreover, the total amount of power on multiplexed subcarriers is deducted from the power limit on each constrained antenna. In other terms, the new power limit on the $i^{\text {th }}$ power-constrained RRH is reduced to:

$$
P_{m_{i}}^{\prime}=P_{m_{i}}-\sum_{n \in S\left(R L_{i}\right) \text { s.t } m(n)=2} P_{k, n, R L_{i}}
$$

Therefore, a necessary condition to allow the power correction of the system is to prevent any subcarrier pairing that would lead to a total power of multiplexed subcarriers greater than $P_{m_{i}}$ for any antenna in $\mathcal{R} \mathcal{L}$. To keep track of the total multiplexed power on $\mathcal{R} \mathcal{L}$ antennas, we initialize the vector $P_{R L_{i}}$ of $|\mathcal{R} \mathcal{L}|$ elements to zero. For every subcarrier-RRH candidate, the powers $P_{k_{2}, n, r_{2}(n)}$ and $P_{k_{1}, n, r_{1}(n)}$ of the involved users $k_{1}$ and $k_{2}$ are added to their corresponding $P_{R L_{i}}$ elements. If this addition results in an excess on an antenna from $\mathcal{R} \mathcal{L}$, the current candidate pair is denied multiplexing. Meanwhile, the power limit on the multiplexed subcarriers per constrained antenna (i.e. the second term in the left-hand part of (18)) is set to a fraction $\beta(0<\beta<1)$ of $P_{m_{i}}$, in order to leave room for power adjustment (correction). 
Similarly to the orthogonal scenario, the subcarrier pairing must leave at least one sole subcarrier for each user powered by an RRH in $\mathcal{R U}$ in order to guarantee the existence of a solution to the PA problem. This pairing procedure can be coupled with either OMA-HDAS or OMA-HDAS-Realloc. Note that the amounts of power on multiplexed subcarriers are determined using the LPO (local power optimization) and DPA (direct power adjustment) procedures introduced in [14] for single SIC and mutual SIC subcarriers respectively. Finally, the power correction is performed on the sole subcarriers with $P_{m_{i}}^{\prime}$ instead of $P_{m_{i}}$ in (13).

\begin{tabular}{l}
\hline Algorithm 3 NOMA-HDAS \& NOMA-HDAS-Realloc \\
Initialization: $\mathcal{U}_{0}=\{1,2, \ldots, K\} ; \mathcal{S}_{k}=\emptyset, \forall k \in \mathcal{U}_{0} ; \mathcal{S}_{p}=\{1,2, \ldots, S\}$
\end{tabular}

Phase 1:

Apply OMA-HDAS or OMA-HDAS-Realloc without power correction

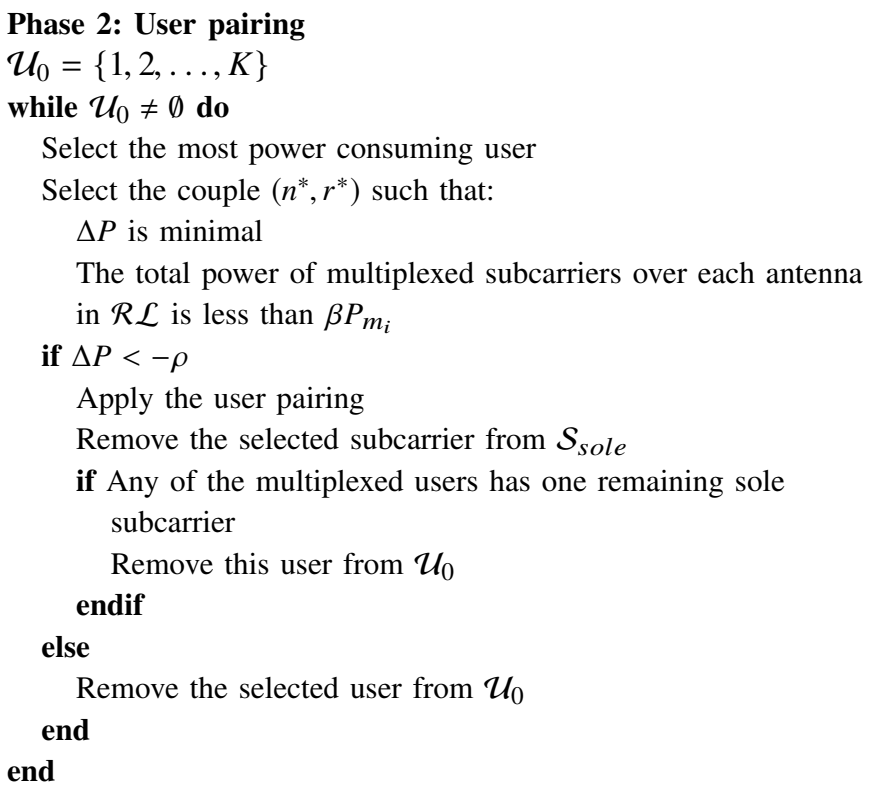

Phase 3: Power correction

if $P_{\mathcal{R} \mathcal{L}}>P_{m}$ Apply the power correction on $\mathcal{S}_{\text {sole }}$

end

\section{COMPLEXITY ANALYSIS}

In this section, we assess the complexity of the proposed resource allocation techniques. Given that the algorithms consist in sequential blocks of OMA assignment, OMA reallocation, and NOMA pairing, we analyze the complexity of each independent step and then derive the complexity of each algorithm by combining the corresponding steps.

In the OMA section, the core of the algorithm resides in searching for the most power consuming user, which presents a linear complexity with the number of users $(O(K))$, assigning to him the best subcarrier-RRH pair $(O(R S))$, and reiterating this process $S$ times until all subcarriers are allocated. The resulting complexity is of $O(S(K+R S))$. Suppose, on the other hand, that we proceed to sorting the $R \times K$ vectors of subcarriers (of length $S$ ) in the channel matrix $\mathbb{H}$ prior to subcarrier-RRH assignment. In other words, the channel matrix is rearranged in a way that the subcarriers of each user are sorted by the decreasing order of channel gain, separately for each RRH. In this case, the assignment, to the selected user, of the best available subcarrier-RRH pair reduces to searching for the best antenna with a complexity linear with $R$. Sorting $\mathbb{H}$ reduces the complexity of the subcarrier-RRH allocation phase $S$ times, while adding a sorting complexity of $K S R \log (S)$. Each allocation cycle consists then of user identification, followed by the search of the RRH providing the subcarrier with the highest channel gain. The resulting complexity of this new approach is therefore $O(K S R \log (S)+S(K+R))$. This approach is roughly $S / K \log (S)$ times less complex then the preceding one (without matrix reordering), hence it will be used hereinafter for all the algorithms. The complexity of OMA-HDAS before power correction is in $O(K S R \log (S)+S(K+R))$, and that of OMA-HDAS-Realloc before the reallocation phase is $O(K S(R-F) \log (S)+(K+R-F) S)$. Since the $\mathcal{R} \mathcal{L}$ antennas are not used until the reallocation phase, $\mathbb{H}$ does not need to be sorted on the corresponding $F$ RRHs.

During the reallocation phase, the most power consuming user is first selected $(O(K))$, then its subcarriers are checked for a potential emission from the $\mathcal{R} \mathcal{L}$ antennas. The selected subcarrier satisfies Proposition. 2 which requires determining the best antenna for every candidate subcarrier. Assuming an equal distribution of the number of subcarriers among users, the complexity of reallocating a single subcarrier is $O(K+$ $F S / K)$. For the worst case of $S$ reallocated subcarriers, the resulting complexity is upper-bounded by $O(S(K+S F / K))$.

Finally, the subcarrier pairing step consists of selecting the most power consuming user which costs $O(K)$, then searching for the subcarrier-RRH pair minimizing the total power $(O(S R))$. The process is repeated a maximum of $S$ times leading to a complexity of $O(S(K+S R))$. At last, the power correction phase is carried when needed with a computational cost denoted by $f$, which depends on the numerical solver used to resolve the non-linear system in (13). Table I gives the upper bound to the complexity of each technique.

Table I: Approximate complexity of the different allocation techniques.

\begin{tabular}{|l|c|}
\hline \multicolumn{1}{|c|}{ Technique } & Complexity \\
\hline OMA-HDAS & $O(K S R \log (S)+S(K+R)+f)$ \\
\hline OMA-HDAS-Realloc & $O(K S(R-F) \log (S)+S(K+R-F)$ \\
& $+S(K+S F / K)+f)$ \\
\hline NOMA-HDAS & $O(K S R \log (S)+S(K+R)$ \\
& $+S(K+S R)+f)$ \\
\hline NOMA-HDAS-Realloc & $\begin{array}{c}O(K S(R-F) \log (S)+S(K+R-F) \\
+S(K+S F / K)+S(K+S R)+f)\end{array}$ \\
\hline
\end{tabular}

It can be seen that OMA-HDAS and OMA-HDAS-Realloc present similar complexities, since the computational cost of the reallocation phase is compensated by an initial sorting over a smaller user-antenna set of subcarrier vectors. The same 
can be said when comparing the complexity of NOMA-HDAS to that of NOMA-HDAS-Realloc since their NOMA pairing phases are essentially the same. However, when comparing NOMA to OMA algorithms, a noticeable complexity increase can be observed. This is driven by the dominant factor $S^{2} R$ as opposed to the $S^{2} F / K$ term in the reallocation phase. Since the cost of the power correction is the same for all techniques, we compare the relatives complexities of the algorithms before power correction. For the configuration of Fig. 3, that is $K=38$ users, $R=4$ RRHs, $S=64$ subcarriers, $F=1$ constrained RRH and $R_{r e q}=5$ Mbps per user, OMA-HDASRealloc is $17.7 \%$ less complex than OMA-HDAS, while NOMA-HDAS is $1.7 \%$ less complex than NOMA-HDASRealloc. Both NOMA techniques are about $46 \%$ more complex than OMA-HDAS.

\section{PERformance EVAluation}

The performance of the proposed resource allocation schemes are assessed for various conditions of antenna power limits, rate requirements and number of users in the cell. Reported simulation results are averaged over 10,000 iterations of various user distributions. Results are compared against OMA-DAS and NOMA-DAS which represent the OMA and NOMA algorithms elaborated in [14] when no power-limits are considered. The system is simulated using a hexagonal cell model with an outer radius $r_{d}$ of $500 \mathrm{~m}$. The network topology consists of four RRHs distributed as follows: one central antenna and three antennas uniformly positioned on a circle of radius $2 r_{d} / 3$ centered at the cell center. In all the simulated scenarios, the central antenna is considered to have no power limitation. In all the figures, except for Fig. 5, a single antenna (randomly chosen) is power limited $(F=1)$, whereas in Fig. 5, one, two and three power limited antennas are considered. Users are randomly deployed in the cell. The transmission medium is a frequency-selective Rayleigh fading channel with a root mean square delay spread $\tau$ of $500 \mathrm{~ns}$. Large scale fading is composed of path-loss with a decay factor of 3.76, and lognormal shadowing with an $8 \mathrm{~dB}$ variance. The system bandwidth $B$ is $10 \mathrm{MHz}$, and is divided into $S=64$ subcarriers. The noise power spectral density $N_{0}$ is $-173 \mathrm{dBm} / \mathrm{Hz}$ and the power threshold $\rho$ is set to $0.01 \mathrm{~W}$.

The power margin $\beta$ is an important parameter in NOMA algorithms. It is essential to ensure the success-guaranteed nature of the algorithms since the remaining power $P_{m_{i}}^{\prime}$ is the actual one being used to solve (13). The cost of the power correction does not truly depend on how distant the actual power of each antenna $i$ is from $P_{m_{i}}^{\prime}$ (i.e. $\left|P_{R L_{i}}-P_{m_{i}}^{\prime}\right|$ ), but rather, it depends greatly on the ratio of $P_{R L_{i}}$ before correction to the effective power limit of the antenna $P_{m_{i}}^{\prime}$. Therefore, a power excess of $1 \mathrm{~W}$ when $P_{m_{i}}^{\prime}=5 \mathrm{~W}$, incurs a much more graceful degradation compared to the case when $P_{m_{i}}^{\prime}=0.01 \mathrm{~W}$. Also, whether the pairing steps leave a high or low amount of power margin with respect to $P_{m_{i}}^{\prime}$ is entirely linked to the randomness of the channel realizations. To counter that, the power margin factor $\beta$ is used to ensure $P_{m_{i}}^{\prime}>(1-\beta) P_{m_{i}}$. The larger the $\beta$, the greater the risk of having a significant power correction toll. Conversely, the lower the $\beta$, the smaller the number of accepted subcarriers for multiplexing, and the smaller the power reduction observed between OMA and NOMA. The optimal value of $\beta$ comes then as a tradeoff in order to minimize the total system power. This optimal value depends on the system parameters, e.g. the targeted rate, the number of users, etc. Nevertheless, practical tests show that any value of $\beta$ between 0.7 and 0.8 always guarantees a near-optimal tradeoff by leaving enough room to $P_{m_{i}}$ for power correction without hindering the pairing process. For this reason, a value of $\beta=0.75$ is chosen in this study.

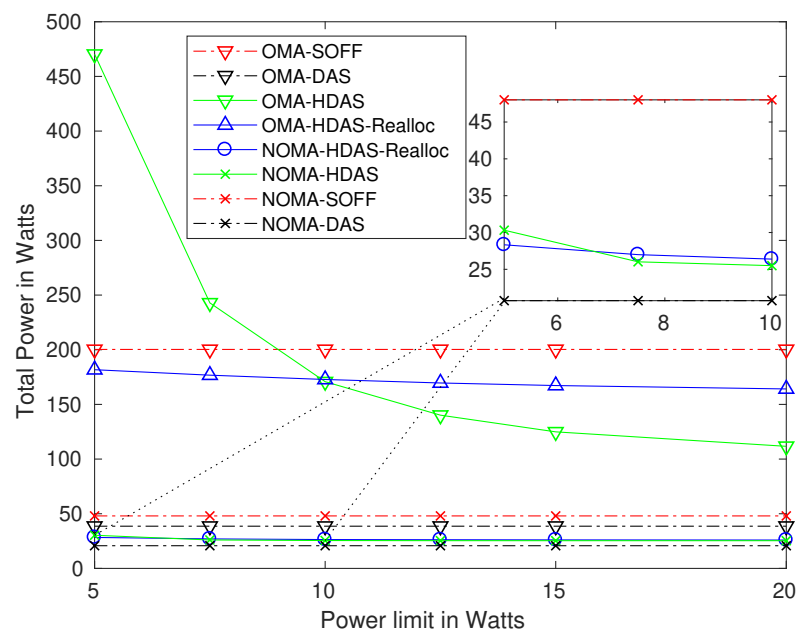

Figure 3: Total power in terms of the antenna power limit for OMA and NOMA schemes, $R_{r e q}=5 \mathrm{Mbps}, K=38$

Fig. 3 represents the total transmit power in the cell as a function of the power limit $P_{m}$. At first, we note the important gap between orthogonal and non-orthogonal RA schemes in which the worst performing NOMA algorithm requires at least $40 \mathrm{~W}$ less power than any other OMA scheme at a power limit of $20 \mathrm{~W}$, to provide all 38 users with a requested rate of $5 \mathrm{Mbps}$. This amounts to a power decrease by more than a factor of two, which means that the complexity increase due to NOMA is largely overcome by the important power savings achieved over OMA. The performance of the algorithms under high power limit constraints gives an indication about the best performance that can be reached by each considered allocation technique. Therefore, in light of this remark and regarding orthogonal RA schemes, OMA-HDAS has a greater potential in limiting system power than OMA-HDAS-Realloc. However, OMA-HDAS only achieves this potential for relatively relaxed power constraints. Also, OMA-HDAS performance deteriorates drastically for more severe conditions: as the power limit decreases, OMA-HDAS leads to an increasingly more important power correction cost until it eventually gets worse than the trivial OMA-SOFF solution in which constrained antennas are simply shut off and the algorithm is run using the remaining antennas. On the other hand, OMA-HDAS-Realloc handles critical power conditions in a much more graceful way. Indeed, its total transmit power remains a reasonably better alternative than the trivial solution, while slightly increasing with the decreasing power limit. This is in accordance with 
the properties that were required from OMA-HDAS-Realloc in providing a solution that always outperforms the trivial solution.

As a conclusion, OMA-HDAS-Realloc performs better OMA by far for critical system conditions, whereas OMAHDAS is better for the other extreme (i.e for loose system conditions of power limit, user rates and number of users). The same analysis can be drawn from the two competing NOMA algorithms as they suffer/benefit from the same advantages/drawbacks as shown in Figs. 3,4. The reason for this behavior being that each NOMA scheme is based on its orthogonal counterpart.

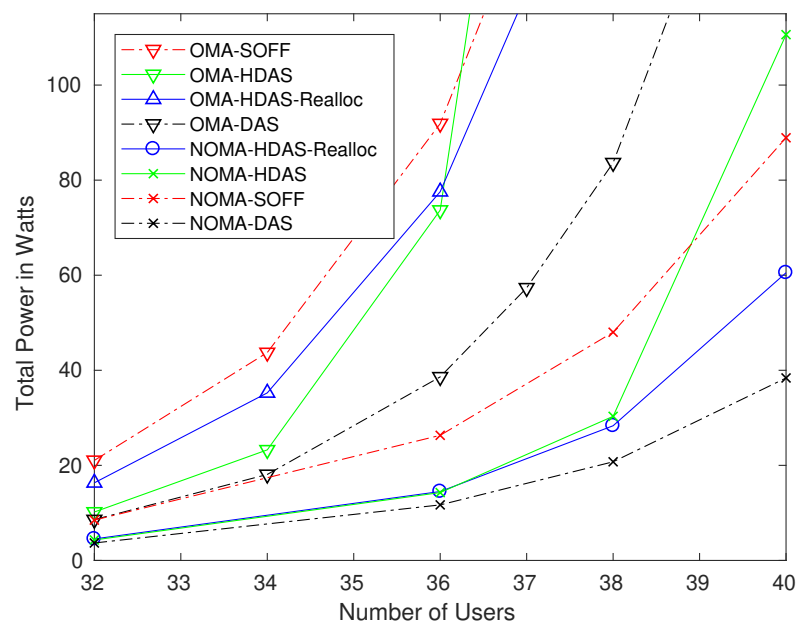

Figure 4: Total power in terms of the number of users for a requested rate of $R_{\text {req }}=5 \mathrm{Mbps}$ with $P_{m}=5 \mathrm{~W}$

In Fig. 4, the performance of the OMA and NOMA schemes are presented as a function of the number of users. It can be observed that the behavior of the NOMA algorithms follows the lead of their OMA counterparts: starting from forgiving or mild system conditions (i.e. for a relatively small number of users), NOMA-HDAS has barely an advantage over NOMA-HDAS-Realloc (14.3 W vs $14.5 \mathrm{~W}$ respectively for 32 users), till the point where the system conditions start to weigh too heavily on NOMA-HDAS, forcing important power corrections. The latter switches the balance in favor of NOMAHDAS-Realloc which requires a transmit power of $60.5 \mathrm{~W}$ for a total of 40 users, incurring a $58 \%$ power increase with respect to NOMA-DAS against $188 \%$ inferred by NOMA-HDAS.

The percentage power increase of NOMA-HDAS-Realloc compared to NOMA-DAS increases with the number of users: $24 \%$ for 36 users, $37 \%$ for 38 users, and $58 \%$ for 40 users. This increase is expected since the total system power is increasing with the number of users while the imposed power-limit remains unchanged. Finally, we note the important reduction in the performance gaps when moving from OMA to NOMA, between HDAS-Realloc and HDAS algorithms, within the regions of forgiving or mild system conditions. For example, for 32 users, a relative power difference of $60 \%$ is observed in the orthogonal context vs $5 \%$ of difference in the non-orthogonal one. This convergence of the algorithms in regions previously favorable to NOMA-HDAS promotes NOMA-HDAS-Realloc as a globally better candidate for resolving our RA problem.

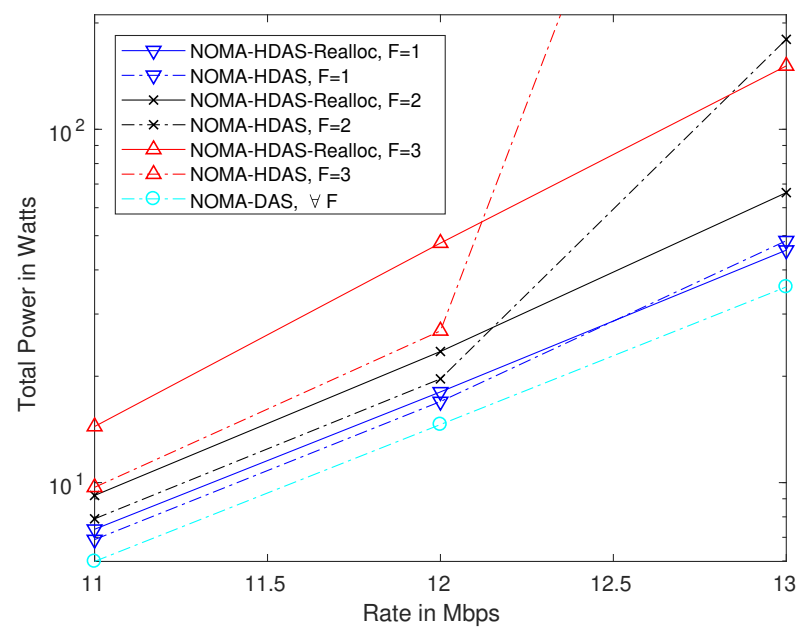

Figure 5: Total power in terms of the target rate, for different values of the number of constrained antennas, with $K=15$ and $P_{m_{i}}=15 \mathrm{~W}$

Fig. 5 shows the evolution of the system power with the number of constrained antennas. As expected, the greater the number of constrained antennas, the more important the total power. Moreover, when comparing NOMA-HDAS and NOMA-HDAS-Realloc at $13 \mathrm{Mbps}$, we observe that the correction costs increase with the number of constrained antennas. At a rate of $13 \mathrm{Mbps}$, the difference between NOMA-HDAS and NOMA-HDAS-Realloc, is $0.27 \mathrm{~dB}(6.4 \%), 4.3 \mathrm{~dB}(169 \%)$ and $18.3 \mathrm{~dB}(6660 \%)$ for $F=1,2$ and 3 respectively. However, at lower values of the requested rate, the saved power of NOMA-HDAS with respect to NOMA-HDAS-Realloc is even larger for a larger number of constrained antennas $(0.27 \mathrm{~dB}$ (6.4\%), $0.77 \mathrm{~dB}(19.4 \%)$ and $2.5 \mathrm{~dB}(77.8 \%)$ for $F=1,2$ and 3 respectively at $12 \mathrm{Mbps})$. We conclude that the increase in the number of constrained antennas magnifies the differences between NOMA-HDAS-Realloc and NOMA-HDAS, both in critical and mild conditions.

\section{CONCLUSION}

In this study, we addressed the problem of power minimization under user rate requirements and antenna power limit considerations in a hybrid DAS scenario. We first explored the characteristics of optimum power allocation in an orthogonal scenario, which enabled the design of RA schemes for both orthogonal and non-orthogonal contexts. The results suggest the use of different algorithms depending on system conditions: NOMA-HDAS is favored in the presence of low requested rate, high power limits and small numbers of served users and constrained antennas. On the other hand, NOMA-HDASRealloc prove to be remarkably efficient in harsher system constraints, maintaining a significant advantage over the trivial solution of shutting down the constrained antennas. Thus, relying on a judicious antenna allocation in the first place is preferable over resorting systematically to the optimum power correction procedure. 


\section{ACKNOWLEDGMENT}

This work has been funded with support from the Lebanese University and the PHC CEDRE program (research project between the Lebanese University and IMT Atlantique).

\section{APPENDIX A}

Proof. We calculate the partial derivatives of the Lagrangian in (9) with respect to the power variables. Depending on the RRH powering the subcarrier, there are two possible cases:

- The subcarrier is powered by a power limited antenna - The subcarrier is powered by a non-constrained antenna. For the subcarriers falling in the first category, we have:

$$
\begin{gathered}
\frac{\partial L}{\partial P_{k, n, r(n)}}=0 \\
-1-\frac{B \lambda_{k}}{S \ln (2)} \frac{h_{k, n, r(n)}^{2} / \sigma^{2}}{1+P_{k, n, r(n)} h_{k, n, r(n)}^{2} / \sigma^{2}}-\alpha_{i}=0
\end{gathered}
$$

By setting $m_{k}=-B \lambda_{k} / S \ln (2)$, we get:

$$
\begin{gathered}
-1+\frac{m_{k} h_{k, n, r(n)}^{2} / \sigma^{2}}{1+P_{k, n, r(n)} h_{k, n, r(n)}^{2} / \sigma^{2}}-\alpha_{i} \\
\frac{m_{k}}{\frac{\sigma^{2}}{h_{k, n, r(n)}^{2}}+P_{k, n, r(n)}}=1+\alpha_{i}
\end{gathered}
$$

The subcarriers that are not powered by a constrained antenna do not feature an $\alpha_{i}$ term as in (19). Instead, their partial derivative yields:

$$
\frac{m_{k}}{\frac{\sigma^{2}}{h_{k, n, r(n)}^{2}}+P_{k, n, r(n)}}=1
$$

Equations (19) and (20) lead directly to (10) and (11). This concludes our proof.

\section{APPENDIX B}

Proof. In the case where the subcarriers of a user $k$ are all powered by non-constrained antennas, the solution of the power minimization problem of user $k$ under its rate constraint $R_{k}$ is a classical waterfilling [13]. Let $W_{k}$ be the waterline of user $k$. The power allocated on each subcarrier of $\mathcal{S}_{k}$ is:

$$
P_{k, n, r(n)}=W_{k}-\frac{\sigma^{2}}{h_{k, n, r(n)}^{2}}
$$

By injecting (21) into the rate constraint (7), we obtain:

$$
\begin{gathered}
\sum_{n \in \mathcal{S}_{k}} \frac{B}{S} \log _{2}\left(\frac{W_{k} h_{k, n, r(n)}^{2}}{\sigma^{2}}\right)=R_{k} \\
\frac{B}{S} \log _{2}\left(W_{k}^{\left|\mathcal{S}_{k}\right|} \prod_{n \in \mathcal{S}_{k}} \frac{h_{k, n, r(n)}^{2}}{\sigma^{2}}\right)=R_{k}
\end{gathered}
$$

Therefore:

$$
W_{k}=\left(\frac{2^{R_{k} S / B}}{\prod_{n \in \mathcal{S}_{k}} \frac{h_{k, n, r(n)}^{2}}{\sigma^{2}}}\right)^{1 /\left|\mathcal{S}_{k}\right|}
$$

This expression of $W_{k}$ directly leads to the relationship (12).

\section{APPENDIX C}

Proof. Based on the expression of the waterline of user $k$ (equation (22) from Appendix B), the new waterlevel obtained from the reallocation of subcarrier $n$ to $r_{n e w}$ can be expressed as:

$$
W_{k, \text { new }}=W_{k}\left(\frac{h_{\text {old }}}{h_{\text {new }}}\right)^{2 /\left|S_{k}\right|}
$$

The power variation of user $k$ obtained from this potential reallocation is:

$$
\Delta P=\left|\mathcal{S}_{k}\right|\left(W_{k, \text { new }}-W_{k}\right)
$$

The subcarrier to be selected must verify:

$$
n^{*}=\underset{n \in \mathcal{S}_{k}}{\arg \min } \Delta P=\underset{n \in \mathcal{S}_{k}}{\arg \min } W_{k, \text { new }}=\underset{n \in \mathcal{S}_{k}}{\arg \max } \frac{h_{\text {new }}}{h_{\text {old }}}
$$

Then, it is straightforward that the selected RRH should be $r_{\text {new }}=\underset{r \in \mathcal{R} \mathcal{L}}{\arg \max }\left(h_{k, n^{*}, r}\right)$. This concludes our proof.

\section{REFERENCES}

[1] R. Heath, S. Peters, Y. Wang, and J. Zhang, "A Current Perspective on Distributed Antenna Systems for the Downlink of Cellular Systems," IEEE Commun. Mag., vol. 51, no. 4, pp. 161-167, Apr 2013.

[2] Z. Liu and L. Dai, "A Comparative Study of Downlink MIMO Cellular Networks With Co-Located and Distributed Base-Station Antennas," IEEE Trans. Wireless Commun., vol. 13, no. 11, pp. 6259-6274, Nov 2014.

[3] W. Choi and J. G. Andrews, "Downlink Performance and Capacity of Distributed Antenna Systems in a Multicell Environment," IEEE Trans. Wireless Commun., vol. 6, no. 1, pp. 69-73, Jan 2007.

[4] R. W. H. Jr, T. Wu, Y. H. Kwon, and A. C. K. Soong, "Multiuser MIMO in Distributed Antenna Systems With Out-of-Cell Interference," IEEE Trans. Signal Process., vol. 59, no. 10, pp. 4885-4899, Oct 2011.

[5] F. Yuan, S. Jin, Y. Huang, K. k. Wong, Q. T. Zhang, and H. Zhu, "Joint Wireless Information and Energy Transfer in Massive Distributed Antenna Systems," IEEE Commun. Mag., vol. 53, no. 6, pp. 109-116, Jun 2015.

[6] H. Ren, N. Liu, C. Pan, and C. He, "Energy Efficiency Optimization for MIMO Distributed Antenna Systems," IEEE Transactions on Vehicular Technology, vol. 66, no. 3, pp. 2276-2288, March 2017.

[7] C. He, G. Y. Li, F. C. Zheng, and X. You, "Energy-Efficient Resource Allocation in OFDM Systems with Distributed Antennas," IEEE Trans. on Veh. Technol., vol. 63, no. 3, pp. 1223-1231, Mar 2014.

[8] M. Tham, S. F. Chien, D. W. Holtby, and S. Alimov, "Energy-Efficient Power Allocation for Distributed Antenna Systems With Proportional Fairness," IEEE Trans. on Green Commun. and Netw., vol. 1, no. 2, pp. 145-157, June 2017.

[9] L. Dai, B. Wang, Y. Yuan, S. Han, C. I, and Z. Wang, "Non-orthogonal Multiple Access for 5G: solutions, challenges, opportunities, and future research trends," IEEE Commun. Mag., vol. 53, no. 9, pp. 74-81, September 2015.

[10] Y. Saito, Y. Kishiyama, A. Benjebbour, T. Nakamura, A. Li, and K. Higuchi, "Non-Orthogonal Multiple Access (NOMA) for Cellular Future Radio Access," in 2013 IEEE 77th Veh. Technol. Conf. (VTC Spring), June 2013, pp. 1-5.

[11] Z. Ding, Z. Yang, P. Fan, and H. V. Poor, "On the Performance of NonOrthogonal Multiple Access in 5G Systems with Randomly Deployed Users," IEEE Signal Process. Lett., vol. 21, no. 12, pp. 1501-1505, Dec 2014.

[12] Y. Saito, A. Benjebbour, Y. Kishiyama, and T. Nakamura, "System-Level Performance Evaluation of Downlink Non-Orthogonal Multiple Access (NOMA)," in 2013 IEEE 24th Annual Int. Symp. Personal, Indoor, and Mobile Radio Comm. (PIMRC), Sep 2013, pp. 611-615.

[13] J. Farah, E. Sfeir, C. A. Nour, and C. Douillard, "New Resource Allocation Techniques for Base Station Power Reduction in Orthogonal and Non-Orthogonal Multiplexing Systems," in 2017 IEEE Int. Conf. on Comm. Workshops (ICC Workshops), May 2017, pp. 618-624. 
[14] J. Farah, A. Kilzi, C. A. Nour, and C. Douillard, "Power Minimization in Distributed Antenna Systems using Non-Orthogonal Multiple Access and Mutual Successive Interference Cancellation," IEEE Trans. Veh. Technol., in press.

[15] W. Choi and J. G. Andrews, "Downlink Performance and Capacity of Distributed Antenna Systems in a Multicell Environment," IEEE Trans. on Wireless Commun., vol. 6, no. 1, pp. 69-73, Jan 2007.

[16] M.-R. Hojeij, J. Farah, C. A. Nour, and C. Douillard, "New Optimal and Suboptimal Resource Allocation Techniques for Downlink NonOrthogonal Multiple Access," Wireless Personal Commun., vol. 87, no. 3, pp. 837-867, Apr 2016.

[17] D. D. Yu and J. M. Cioffi, "SPC10-2: Iterative Water-filling for Optimal Resource Allocation in OFDM Multiple-Access and Broadcast Channels," in IEEE Globecom 2006, Nov 2006, pp. 1-5.

[18] J. Farah, E. Sfeir, C. A. Nour, and C. Douillard, "New Efficient EnergySaving Techniques for Resource Allocation in Downlink OFDMA Transmission Systems," in 2017 IEEE Symp. on Comput. and Commun. (ISCC), July 2017, pp. 1056-1062.

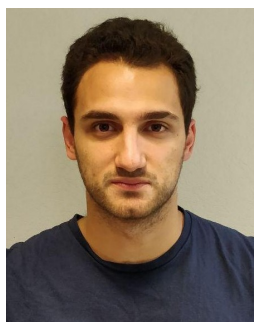

Antoine Kilzi Antoine Kilzi received his computer and communications engineering degree in 2017 from the Lebanese University. He is currently working towards the Ph.D. degree in information and communication engineering at IMT Atlantique. His current research interests include resource allocation, non-orthogonal multiple access and coordinated multipoint systems.

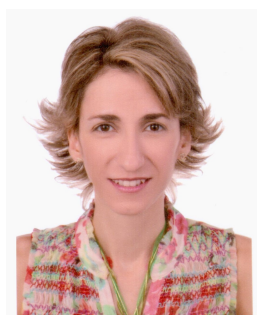

Joumana Farah Joumana Farah received the B.E degree in Electrical Engineering from the Lebanese University, in 1998, the M.E. degree in Signal, Image, and Speech processing, in 1999, and the $\mathrm{Ph} . \mathrm{D}$. degree in mobile communication systems, in 2002, from the University of Grenoble, France. In 2010, she received the Habilitation to Direct Research (HDR) from University Pierre and Marie Curie (Paris VI), France. She is currently a fulltime professor with the Faculty of Engineering, Lebanese University, Lebanon. She has supervised a large number of Master and $\mathrm{PhD}$ theses. She has received several research grants from the Lebanese National Council for Scientific Research, the Franco-Lebanese CEDRE program, and the Lebanese University. She has nine registered patents and a software and has coauthored a research book and a hundred of papers in international journals and conferences. Her current research interests include resource allocation techniques, channel coding, channel estimation, interference management, and distributed video coding. She was the General Chair of the 19th International Conference on Telecommunications (ICT 2012), and serves as a TPC member and a reviewer for several journals and conferences.

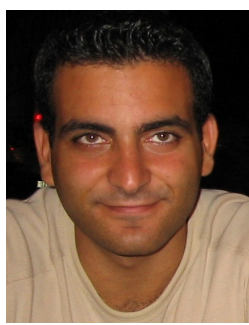

Charbel Abdel Nour Charbel Abdel Nour obtained his computer and communications engineering degree in 2002 from the Lebanese University, his Masters degree in digital communications from the University of Valenciennes, France, in 2003 and his $\mathrm{PhD}$ in digital communications from Telecom Bretagne, France in 2008. From June 2007 till October 2011, he worked as a post-doctoral fellow at the Electronics Department of Telecom Bretagne. He was involved in several research projects related to broadcasting and satellite communications. Additionally during the same period, he was active in the Digital Video Broadcasting DVB consortium where he had important contributions. Starting November 2011, Charbel holds an associate professor position at the Electronics Department of Telecom Bretagne. His interests concern the radio mobile communications systems, broadcasting systems, coded modulations, error correcting codes, resource and power allocation for NOMA, waveform design, MIMO and iterative receivers. Lately, he presented several contributions to the H2020 METIS and FANTASTIC5G projects and to the 3GPP consortium related to coding solutions for $5 \mathrm{G}$.

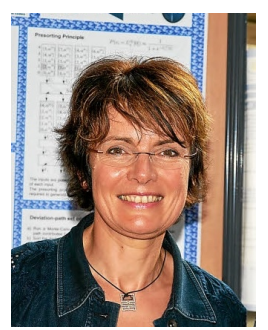

Catherine Douillard Catherine Douillard received the engineering degree in telecommunications from the Ecole Nationale Supérieure des Télécommunications de Bretagne, France, in 1988, the $\mathrm{Ph} . \mathrm{D}$. degree in electrical engineering from the University of Western Brittany, Brest, France, in 1992, and the accreditation to supervise research from the University of Southern Brittany, Lorient, France, in 2004. She is currently a full Professor in the Electronics Department of Telecom Bretagne where she is in charge of the Algorithm-Silicon Interaction research team. Her main research interests are turbo codes and iterative decoding, iterative detection, the efficient combination of high spectral efficiency modulation and turbo coding schemes, diversity techniques and turbo processing for multi-carrier, multi-antenna and multiple access transmission systems. In 2009, she received the SEE/IEEE Glavieux Award for her contribution to standards and related industrial impact. She was active in the DVB (Digital Video Broadcasting) Technical Modules for the definition of DVB-T2, DVB-NGH as chairperson of the "Coding, Constellations and Interleaving" task force and DVB-RCS NG standards. Since 2015, she has had several contributions in the FANTASTIC-5G and EPIC H2020 European projects intended for the definition of new techniques for $5 \mathrm{G}$ and beyond. 